\title{
Paraneoplastic Encephalomyelitis and Subacute Dysautonomia due to an Occult Atypical Carcinoid Tumour of the Lung
}

\author{
M. Veilleux, J.P. Bernier, and J.B. Lamarche
}

\begin{abstract}
A case of paraneoplastic encephalomyelitis and subacute pandysautonomia associated with an occult atypical carcinoid tumour of the lung is described. The main clinical features were lethargy, impaired memory, constipation, and orthostatic hypotension. Neurological investigation was unremarkable except for mononuclear pleocytosis and increased protein level in the cerebrospinal fluid (CSF). Tests of autonomic function revealed a low plasma norepinephrine level, a marked drop of blood pressure (BP) to vertical tilt and Valsalva maneuver, and a marked rise of BP to dilute norepinephrine infusion. A few days prior to death, the patient became hypothermic and had repeated episodes of respiratory arrest associated with transient atrioventricular block on the electrocardiogram (ECG). A polysomnographic study confirmed a sleep apnea syndrome. Autopsy revealed an atypical carcinoid tumour in one tracheobronchial lymph node, widespread lymphocytic infiltrates and loss of neurones in the cerebral, cerebellar and brainstem gray matter, the spinal cord and roots, and the paravertebral sympathetic ganglia as well as microglial and astrocytic proliferation in the central nervous system.
\end{abstract}

RÉSUMÉ: Encéphalomyélite paranéoplasique et dysautonomie subaiguë dues à une tumeur carcinoïde atypique occulte du poumon Nous décrivons un cas d'encéphalomyélite paranéoplasique et de pandysautonomie subaiguë associées à une tumeur carcinoïde atypique occulte du poumon. Les manifestations cliniques principales étaient de la létargie, un déficit mnésique, de la constipation et de l'hypotension orthostatique. L'investigation neurologique était sans particularité sauf pour une pléocytose mononucléaire et une augmentation du taux de protéines dans le liquide céphalo-rachidien. Les épreuves de fonction autonome ont montré un taux abaissé de norépinéphrine plasmatique, une chute importante de la tension artérielle (TA) à la bascule verticale et à la manoeuvre de Valsalva, et une élévation marquée de la TA à l'infusion d'une solution de norépinéphrine. Quelques jours avant le décès, le patient a présenté de l'hypothermie et des épisodes répétés d'arrêt respiratoire associés à un bloc auriculo-ventriculaire transitoire à l'électrocardiogramme. Une étude polysomnographique a confirmé la présence d'un syndrome d'apnée du sommeil. L'autopsie a montré une tumeur carcinoïde atypique dans un ganglion lymphatique trachéo-bronchique, des infiltrats lymphocytaires étendus et une perte de neurones dans la substance grise cérébrale, cérébelleuse et du tronc cérébral, la moelle épiniaire et les racines nerveuses, les ganglions sympathiques paravertébraux ainsi qu'une prolifération microgliale et astrocytaire dans le système nerveux central.

Can. J. Neurol. Sci. 1990, 17:324-328

Paraneoplastic encephalomyelitis has been previously reported in patients with bronchogenic carcinoma and, less frequently, breast, uterine, ovarian, kidney, and gastric carcinomas. 1-8 Subacute paraneoplastic pandysautonomia has been described in a few patients with bronchogenic carcinoma ${ }^{8-12}$ and two patients with a pancreatic carcinoma. ${ }^{14,15}$ However, to our knowledge, both paraneoplastic conditions have not been reported in the same individual. A case of encephalomyelitis and subacute pandysautonomia associated with an occult atypical carcinoid tumour of the lung is described. This case also showed clinical evidence of a sleep apnea syndrome.

\section{Case Report}

A 72-year-old man was admitted to the hospital in January 1981 with a 6-month history of fatigue, weight loss of 20-30 pounds, constipation, and dizziness on standing upright. In the month prior to admission, he could not stand up and he felt faint or lost consciousness upon standing. In addition, he had urinary retention that required intermittent

From the Department of Neurology (M.V.), Montreal General Hospital and McGill University, and Department of Neurology (J.P.B.) and Pathology (J.B.L.), University of Sherbrooke

Received September 20, 1988. Accepted in final form February 12, 1990

This paper was presented at the Twenty-third Canadian Congress of Neurological Science meeting, Quebec 1988

Reprint requests to: Dr. Martin Veilleux, Division of Neurology, Montreal General Hospital, 1650 Cedar Avenue, Montreal, Quebec, Canada H3G IA4 
catheterization. A definite history of impotence could not be obtained. There was no previous history of diabetes mellitus. He had consumed large quantities of alcohol until 1976, but he had totally abstained thereafter. He had smoked 5-6 cigarettes a day for many years. His only previous illness was a duodenal ulcer treated by vagotomy and gastrojejunostomy in 1979.

Vital signs revealed a blood pressure (BP) which was $90 / 70$ supine and unrecordable upon standing up and a regular heart rate (HR) at $72 /$ minute which did not significantly change when his BP fell. Brief generalized tonic-clonic movements were seen associated with the BP drop. His general examination was unremarkable except for absence of sweating and reduced tearing. Neurological examination was positive only for sluggishly reactive pupils, hypoactive knee and upper limb reflexes, and absent ankle reflexes. Mental status examination revealed a patient who was awake and alert but disoriented to time and place. His spontaneous speech output was markedly reduced. There was no anomia, paraphasia, or grammatical or syntactic errors. He could correctly execute simple mental arithmetic. His recent memory was severely impaired but distant memory was relatively well preserved. He was apathetic and showed no concern about his illness. No formal neuropsychological testing was performed.

Investigations - The following laboratory data were normal: complete blood count, serum electrolytes, serum osmolarity, SMA-12 profile, thyroid function tests, serum cortisol, intravenous ACTH infusion test and measure of cortisol response, FSH and LH plasma level, aldosterone plasma level, serum testosterone, and urinary catecholamines. The ECG revealed a sinus bradycardia at $55 /$ minute. Chest $x$-rays were normal. Barium-meal examination was normal except for evidence of partial gastrectomy and gastrojejunostomy. The CSF contained 25 cells $/ \mathrm{mm}^{3}$ (100\% mononuclear) and $96 \mathrm{mg} \%$ protein with elevated gamma-globulin at $28 \%$. Smears and culture for CSF were negative for bacteria and fungi, and cytologic examination disclosed no neoplastic cells. Computerized tomography (CT) scan of the head revealed a mild, diffuse cerebral and cerebellar atrophy. The electroencephalogram (EEG), obtained on 6 occasions, showed a progressive deterioration of the background activity. The last 2 EEGs, recorded after cardiorespiratory arrest, were characterized by a marked, diffuse slowing of the background activity, atypical triphasic waves, and a burst-suppression pattern.

Studies of autonomic functions - All autonomic function tests were studied by methods previously described by Bannister et al. 15 Tilt table testing showed a marked fall in BP from $172 / 78$ to $118 / 54 \mathrm{~mm} \mathrm{Hg}$ at a 60 degrees tilt and a slight drop in the HR from 50 to 44 /minute, while the plasma norepinephrine (NE) level rose from 10 to $30 \mu \mathrm{g} / \mathrm{ml}$ [normal response: fall in $\mathrm{BP} \leq 30 / 15 \mathrm{~mm} \mathrm{Hg}$, increase in HR $>10$ beats/minute, and plasma NE increase $>80 \%] .15,17.19$ The cold pressor test showed a reduction of the BP from $164 / 78$ to $108 / 52 \mathrm{~mm} \mathrm{Hg}$ after the hands were put in ice water for 60 seconds [normal response: $\geq 15 \mathrm{~mm} \mathrm{Hg}$ increase in systolic and diastolic BP].17-18 The Valsalva maneuver while supine showed a BP drop from $112 / 72$ to $74 / 38 \mathrm{~mm} \mathrm{Hg}$ with no rebound increase in BP [normal response: initial gradual fall in $\mathrm{BP}$ and a $25 \%$ increase in HR followed by mild systolic overshoot and a $20 \%$ fall in HR].15.17-19 A 3-minute dilute NE infusion $(0.05 \mu \mathrm{g} / \mathrm{kg} / \mathrm{min})$ revealed an increase of the $H R$ from 50 to $70 / \mathrm{min}$ and an increase in the BP from $160 / 80$ to $196 / 96 \mathrm{~mm} \mathrm{Hg}$, while the plasma $\mathrm{NE}$ level rose up to $740 \mu \mathrm{g} / \mathrm{ml}$ (baseline level: $10 \mu \mathrm{g} / \mathrm{ml}$ ) (normal response: $\leq 20 \mathrm{~mm} \mathrm{Hg}$ increase in BP with no HR change or a mild fall in HR]. ${ }^{16-19} \mathrm{An}$ intravenous injection of $0.2 \mathrm{mg}$ atropine resulted in a mild increase of the HR from 50 to $60 / \mathrm{min}$ and a fall of the BP from $172 / 80$ to $132 / 68 \mathrm{~mm} \mathrm{Hg}$ [normal response: $\geq 20 \%$ increase in baseline HR]. ${ }^{17}$

Evolution - The patient's severe orthostatic hypotension was unresponsive to intravenous infusion of $\mathrm{NaCl} 0.9$ at a rate of $100 \mathrm{ml}$ per hour for more than 2 weeks, fludrocortisone $0.1 \mathrm{mg}$ die combined with hydrocortisone $50 \mathrm{mg}$ IV every 8 hours for a period of 12 days, and metoclopramide $10 \mathrm{mg}$ TID for more than 12 days.

Four weeks following admission to hospital, he had repeated episodes of respiratory pauses of 15-20 seconds duration associated with a first-degree atrioventricular block on the ECG. A polysomnographic study revealed frequent obstructive sleep apneas lasting from 15 to 80 seconds. A marked hypothermia was also noted with body temperature varying from 35 to 36 degrees Celsius.

Six weeks following his admission, he had 3 short episodes of cardiorespiratory arrest over a 1 -week period. His neurological condition rapidly deteriorated, and he expired on February 23, 1981 after an illness lasting 8 months.

\section{Pathological Examination}

\section{General pathology}

Postmortem examination was performed 19 hours after death. Pathological findings included bilateral pleural effusion and patchy areas of bronchopneumonia in both lungs. One tracheobronchial lymph node which was found to be enlarged contained a metastatic tumour composed of nests and trabeculae of medium-sized malignant epithelial cells (Figure 1) separated by a delicate fibrovascular stroma. The nucle were generally elongated, contained moderately dense fine chromatin and were frequently arranged in palisades. In general, tumour cells had little, finely granular eosinophilic cytoplasm with indistinct borders. Mitotic figures were very numerous. Mucicarmine stain was negative. Immunoreactivity for pre- and pankeratin was positive. Immunoreactivity for chromogranin and neurofilament was negative. The tumour was diagnosed as an atypical carcinoid tumour. Although a careful dissection of the bronchi and the lung parenchyma failed to disclose a primary tumour, the site of the involved node and the histology of the tumour were highly suggestive of a pulmonary origin.

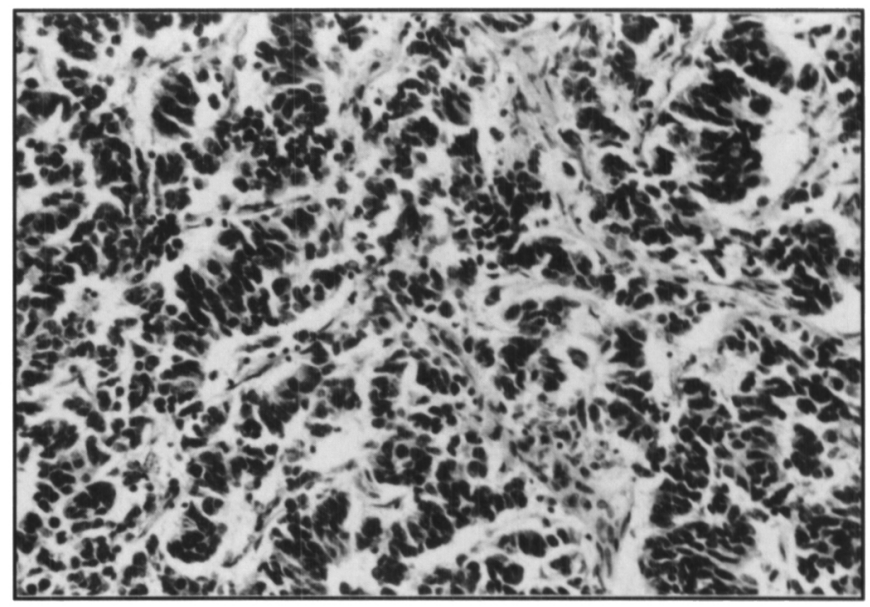

Figure I - Tracheobronchial lymph node with metastatic atypical carcinoid tumour ( $H \& E, x 332)$. Palisading of nuclei is seen.

\section{Neuropathological findings}

The brain weighed $1500 \mathrm{~g}$ after formalin fixation. Macroscopically, the meninges, the brain, the spinal cord, and the paravertebral sympathetic ganglia appeared normal. Cerebral arteries showed minimal atherosclerosis. Coronal sections showed a mild dilatation of the ventricular system. Representative samples of the cerebral cortex, basal ganglia, brainstem, cerebellum, spinal cord, paravertebral autonomic ganglia, and anterior and posterior roots were embedded in paraffin and stained with hematoxylin-eosin, Bodian, Luxol fast blue, and Bodian Congo red stains.

\section{Central Nervous System}

Microscopic examination of the central nervous system revealed a widespread, chronic inflammatory process involving almost exclusively the grey matter, characterized by a subarachnoid and perivascular lymphocytic infiltration, a proliferation and hypertrophy of microglial cells and astrocytes, and a variable neuronal loss. The pathological changes were most prominent in the limbic structures, the brainstem, particularly the medulla oblongata, the cerebellum, and the spinal cord. No inclusion bodies were identified.

In the cerebral neocortex, the corpus striatum and the substantia innominata, there were a few thin perivascular mononuclear cuffs, mild diffuse microglial changes, and a slight neuronal loss associated with a corresponding astrocytic proliferation. In the thalamus, the neuronal depletion was moderate and several degenerating thalamic neurons were also seen. In the hypothalamus, the subcallosal area, the septal nuclei, the amygdaloid nucleus and the end-plate of the hippocampus, 


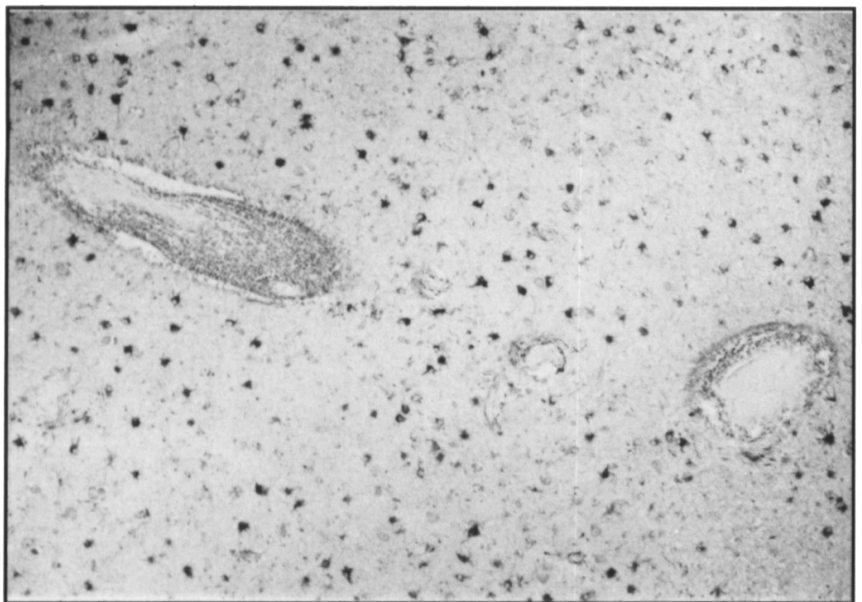

Figure 2 - Section of the amygdaloid complex showing neuronal loss, astrocytic proliferation and perivascular lymphocytic infiltration (GFAP, $x 84$ ).

the perivascular inflammatory cuffs were particularly abundant, and numerous cell nodules were present. In these structures, there was a small number of macrophages, a moderately severe microglial proliferation, and a moderate loss of neurons (Figure 2). Neuronophagia was identified in the end-plate of the hippocampus. With the Bodian Congo red technique, a small number of neurons in the hippocampal formation, the neocortex, and the substantia innominata showed neurofibrillary changes. No senile plaques were detected.

While the perivascular lymphocytic infiltration was inconspicuous in the cerebellar cortex, there was a marked depletion of Purkinje cells associated with a proliferation of Bergmann astrocytes and microglial cells in the molecular layer (Figure 3 ). The remaining Purkinje cells were either normal or atrophic but a few showed pycnotic nuclei and slightly eosinophilic cytoplasm suggestive of acute ischemic changes. Sections of the brainstem showed several dense perivascular cuffs of inflammatory cells, numerous cell nodules, and a moderate to severe neuronal loss in the nuclei of the tegmentum of the pons and the medulla oblongata including the motor dorsal nuclei of the vagus nerve (Figure 4). There was a slight loss of neurons in the locus caeruleus associated with melanin-laden macrophages. In the inferior olivary nuclei, the neuronal depletion was marked, and the microglial hyperplasia and hypertrophy were intense with the formation of microglial nodules.

Sections of the spinal cord showed several dense perivascular cuffs of lymphocytes and cell nodules in the grey matter, especially at the

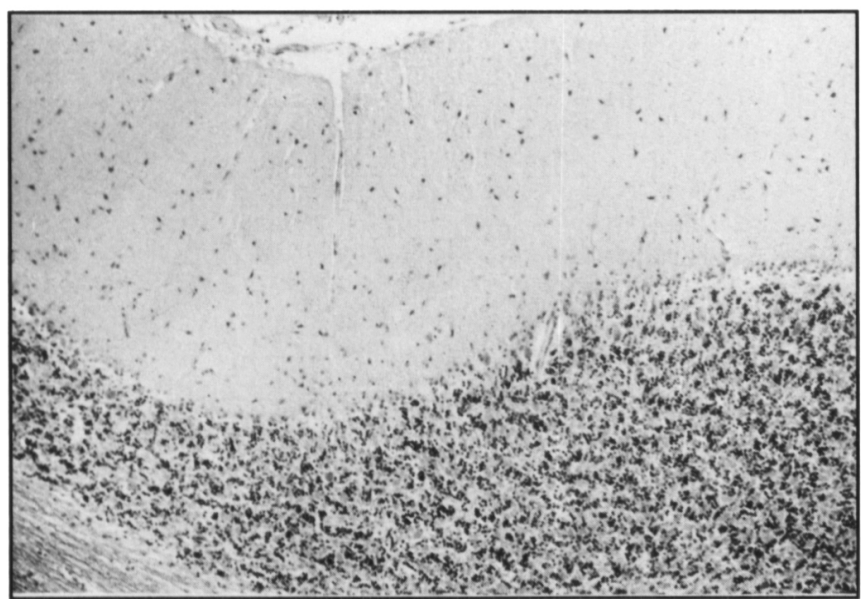

Figure 3 - Section of the cerebellar cortex showing a marked loss of Purkinje cells associated an astrocytic and microglial proliferation $(H \& E, x 85)$.

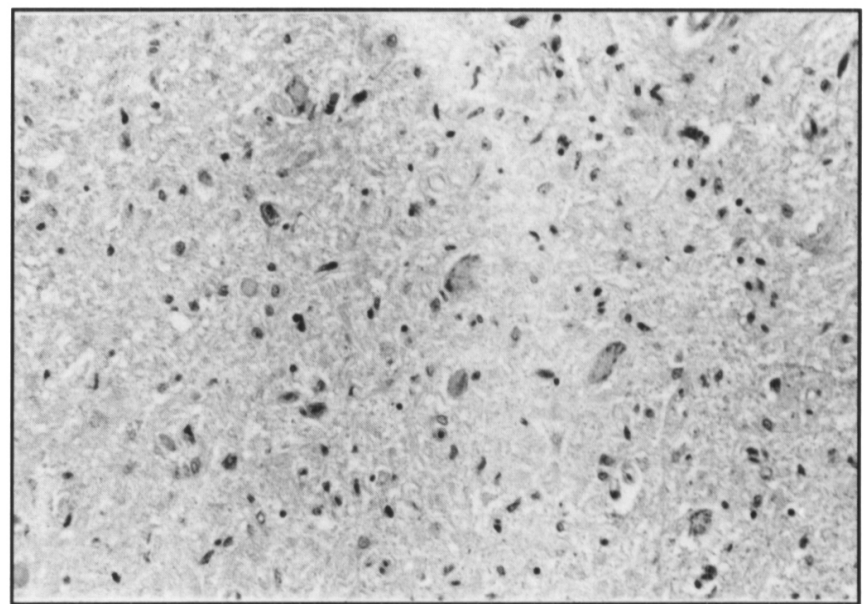

Figure 4-Section of the medulla oblongata showing a loss of neurons, and an astrocytic proliferation in the dorsal motor nuclei of the vagus nerve $(H \& E, \times 212)$.

cervical and thoracic levels. In the anterior and lateral horns, there was also a slight to moderate neuronal loss and the posterior columns, there was a moderate loss of myelinated fibers associated with an active myelin breakdown.

\section{Peripheral Nervous System}

In the anterior and posterior roots, the inflammatory reaction was generally mild with no or only a slight loss of myelinated fibers. In some anterior and posterior thoracic roots, several small perivascular lymphocytic cuffs were found associated with a moderate loss of myelinated fibers and an active myelin breakdown (Figure 5). The dorsal root and paravertebral autonomic ganglia showed a focal interstitial (Figure 6), frequently perivascular, lymphocytic infiltration, a mild to moderate loss of neurons and myelinated fibers with the formation of nodules of Nageotte in the dorsal root ganglia. Neuronophagia was occasionally seen in the autonomic ganglia.

\section{DisCUSSION}

First recognized in 1965 by Henson et al, ${ }^{4}$ the paraneoplastic neurological syndromes include the "limbic" encephalitis and brainstem encephalitis, $2,3,5,7,8$ subacute cerebellar degeneration, $,-8,20$ central 13 and peripheral8-12,21 autonomic dysfunction, myelitis and ganglioradiculitis, $3-8,22$ and the neuromyopathy

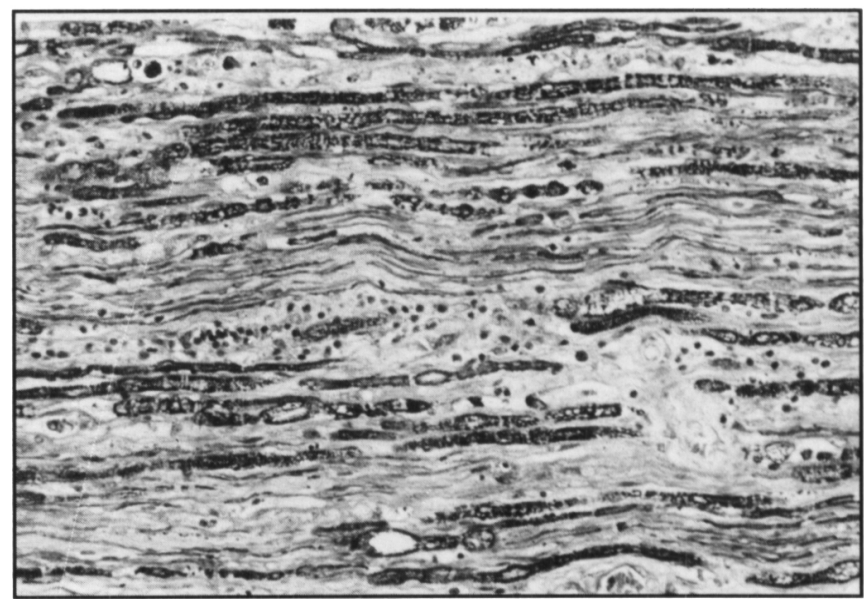

Figure 5 - Section of an anterior thoracic root showing a perivascular lymphocytic infiltration, a loss of large myelinated fibers and degenerated myelin sheets (Luxol fast blue, $x 212$ ). 


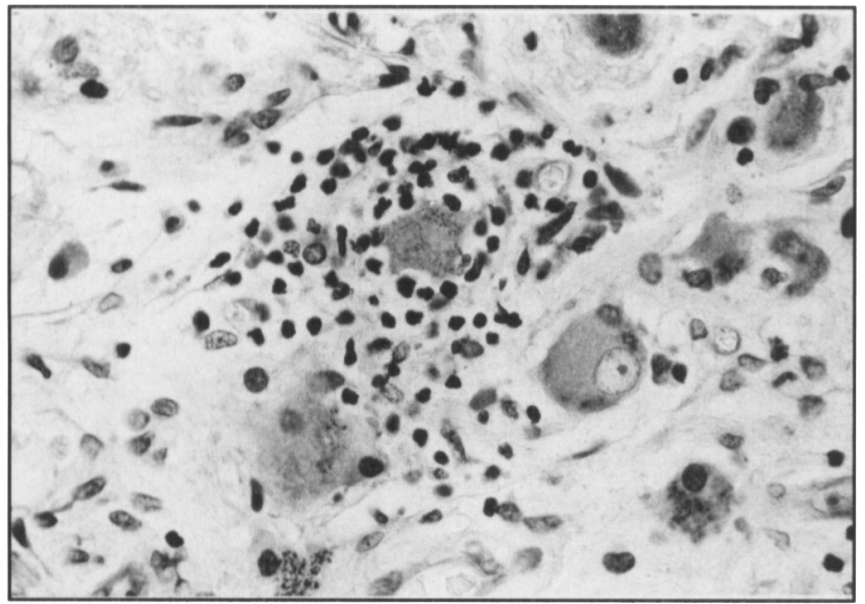

Figure 6-Thoracic sympathetic ganglion showing a lymphocytic infiltration around a degenerating neuron (Lurol fast blue, $x$ 530).

syndromes which comprise among others, the Eaton-Lambert syndrome, the sensorimotor peripheral neuropathy and the polymyositis-dermatomyositis.8,22 The clinical picture in our patient seems unique in that it included a progressive dementia, a pandysautonomia, and a sleep apnea syndrome. The neuropathological findings are also unusual because of the widespread distribution of the chronic inflammatory and degenerative lesions involving the cerebral and cerebellar cortex, the basal ganglia, the brainstem, the autonomic and dorsal root ganglia, and the anterior and posterior roots.

Our patient illustrates the diversity of clinical and pathological findings that characterize the syndrome of paraneoplastic inflammatory degeneration of the nervous system. ${ }^{8}$ One of the salient clinical symptoms was an unexplained apathy, memory deficit, and reduced speech output on mental examination. At autopsy, there were inflammatory and degenerative changes in the thalamus and several limbic structures that could account for the mild, progressive dementia. The pathological changes in the limbic structures were similar to those reported in "limbic" encephalitis associated with carcinoma.2,7,8 Furthermore, in spite of the absence of cerebellar signs on clinical examination, the pathological abnormalities in the cerebellum were similar to those seen in subacute, paraneoplastic cerebellar degeneration. Clinically, the patient had suffered short episodes of cardiorespiratory arrests, and one cannot entirely exclude that hypoxia was the only cause of the severe Purkinje cell loss. This is, however, unlikely as the neurons of the hippocampus and cerebral cortex, two anatomical sites as sensitive to hypoxia were relatively well preserved. Hypoglycemia, another possible etiology for neuronal loss, had never been recorded in the present case.

The patient's most striking clinical symptom was a severe, progressive orthostatic hypotension. Studies of the autonomic functions confirmed an involvement of multiple autonomic structures. The marked increase in BP to dilute NE infusion was consistent with denervation supersensitivity and suggested a lesion of the post-ganglionic sympathetic fibers. ${ }^{15,16,19}$ The presence of a low plasma NE level that failed to significantly increase with standing suggested a lesion in the vasomotor center and a primary failure in activation of the efferent sympathetic limb on standing. ${ }^{15-17,19,24}$ The abnormal cold pressor test was also consistent with involvement of the efferent vasoconstrictor sympathetic fibers. ${ }^{17,19}$ The fall in BP and the absence of BP overshoot during the Valsalva maneuver were suggestive of a lesion of the afferent visceral receptors and the efferent sympathetic pathways. ${ }^{17,19}$ The abnormal heart rate response to Valsalva maneuver pointed to a lesion of the afferent visceral receptors, the cardiac efferent vagal fibers, or their central connections. ${ }^{19}$ The severe hypothermia that occurred late in the course of the disease was due to a lesion of either the central or peripheral sympathetic pathways. ${ }^{25}$ Pathological findings in the hypothalamus, medulla oblongata, spinal cord and roots, and paravertebral sympathetic autonomic ganglia pointed to an involvement of both central and peripheral autonomic structures. Such widespread morphological involvement of the autonomic system made the establishment of precise clinico-pathological correlations impossible. Sympathetic preganglionic fibers may have been injured in several sites including the spinal cord, the anterior root and the paravertebral ganglion whereas post-ganglionic fibers may have been affected in the paravertebral ganglion. Similarly, sympathetic visceral afferent fibers may have been involved in the posterior roots and spinal and paravertebral ganglia. To our knowledge, only Lhermitte et al ${ }^{10}$ also reported similar pathological findings in the autonomic ganglia in a patient who had clinically with a severe orthostatic hypotension and who had a bronchial carcinoma on exploratory thoracotomy. They concluded that the orthostatic hypotension was secondary to the autonomic neuropathy. Chiappa and Young 13 described a case of paraneoplastic pandysautonomia in which a detailed examination of the autonomic system and peripheral nerves revealed no abnormalities.

The third salient clinical feature in our case was a sleep apnea syndrome associated with intermittent first-degree atrioventricular block on the ECG. Pathological examination of the brainstem revealed extensive inflammatory changes with neuronal loss in the pons including the locus caeruleus and in the medulla oblongata. Because most respiratory neurons are located in the reticular formation of the medulla oblongata and centers controlling sleep and upper respiratory tract motility also share the same location, sleep apneas are most likely explained by the pathological changes found in the medulla. Dietl et al ${ }^{22}$ came to a similar conclusion in a case of paraneoplastic brainstem encephalitis associated with central hypoventilation. However, because of destructive lesions in the locus caeruleus in the absence of any major lesions of the medulla oblongata, Kaplan et al ${ }^{6}$ speculated that the degenerative changes in the locus caeruleus were responsible for the central hypoventilation syndrome in a similar case.

The clinical diagnosis of paraneoplastic encephalomyelitis is hampered by the protean manifestations. As pointed out by some authors, $3,6.7$ the diagnosis of encephalomyelitis associated with a remote carcinoma should be suspected in a patient presenting with an unexplained weight loss and a rapidly progressive mental deterioration occurring in middle or late life, with neurological signs suggestive of a neuromuscular disease or a cerebellar degeneration, with elevated protein and lymphocytic pleocytosis in the CSF, with nonspecific EEG abnormalities, and normal or nonspecific findings on head CT scan. Neuropathological examination of the central and peripheral nervous system usually reveals chronic, non specific, inflammatory and degenerative changes affecting only a few or many structures at one or many levels that can account for the diversity in the clinical presentation. A bronchogenic carcinoma, in 
particular a small-cell ("oat-cell") carcinoma, is frequently found in these patients and is not uncommonly occult. In our case, the tumour was histologically intermediate between small cell indifferentiated carcinoma (oat cell) and typical carcinoid tumour, and correspond to the atypical carcinoid tumour reported in the literature. ${ }^{26}$ To our knowledge, this tumour had not been previously reported in association with paraneoplastic manifestations. However, as stated by Mills et al, ${ }^{26}$ this tumour shares similar clinical, neuroendocrine, and pathological features with the small-cell bronchogenic carcinoma and is sometimes confused with it.

The pathogenesis of the paraneoplastic neurological syndromes remains as yet unknown. 5,7,8,20 Three main hypotheses have been proposed. One suggests that the tumour secretes neurotoxic substances. However, no neurotoxins have ever been identified. ${ }^{8} \mathrm{~A}$ second hypothesis proposes that some neurological paraneoplastic syndromes are caused by viral infections. Except for progressive multifocal leukoencephalopathy and possibly subacute motor neuronopathy, it is unlikely that other paraneoplastic neurological syndromes are caused by an infection. The third hypothesis contends that the remote effects of malignant disease on the nervous system result from an autoimmune process. Although the autoimmune hypothesis is by no means proved, antibodies toxic to the nervous system have been found in Lambert-Eaton myasthenic syndrome, paraneoplastic cerebellar degeneration, subacute sensory neuropathy, paraproteinemia with peripheral neuropathy, and paraneoplastic retinal degeneration. ${ }^{8}$ It is also possible that each of these pathogenic mechanisms plays a role in one or more paraneoplastic neurological syndromes.

\section{REFERENCES}

1. Brierley JB, Corsellis JAN, Hierons R, et al. Subacute encephalitis of later life, mainly affecting the limbic areas. Brain 1960; 83: 357-368.

2. Corsellis JAN, Goldberg GJ, Norton AR. "Limbic encephalitis" and its association with carcinoma. Brain 1968; 91: 481-496.

3. Dorfman LJ, Forno LS. Paraneoplastic encephalomyelitis. Acta Neurol Scand 1972; 48: 556-574.

4. Henson RA, Hoffman HL, Urich H. Encephalomyelitis with carcinoma. Brain 1965; 88: 449-464.

5. Henson RA, Urich $\mathrm{H}$. Remote effects of malignant disease: certain intracranial disorders. I $n$ : Vinken PJ, Bruyn GW, eds. Handbook of Clinical Neurology. Amsterdam: Elsevier North-Holland Publishing Co 1979; 38: 625-668.

6. Kaplan AM, Itabashi HH. Encephalitis associated with carcinoma - central hypoventilation syndrome and cytoplasmic inclusion bodies. J Neurol Neurosurg Psychiatry 1974; 37: 1166-1176.

7. Dubas F, Gray F, Escourolle R, et al. Polioencephalomyelites subaigues avec cancer. Rev Neurol 1982; 138 (10): 725-742.

8. Anderson NE, Cunningham JM, Posner JB. Autoimmune pathogenesis of paraneoplastic neurological syndromes. CRC Critical Reviews in Neurobiology 1987; 3: 245-299.

9. Green GJ, Breckenridge AM, Wright FK. Severe hypotension associated with carcinoma of the bronchus. Postgrad Med J 1979; 55 : 426-429.

10. Lhermitte F, Gautier JC, Escourolle R, et al. Hypotension orthostatique et neuropathie périphérique au cours de l'évolution d'un carcinome bronchique. Presse Méd 1970; 78: 257-260.

11. Park DM, Johnson RH, Crean GP, et al. Orthostatic hypotension in bronchial carcinoma. Br Med J 1972; 3: 510-511.

12. Siemsen JK, Meister L. Bronchogenic carcinoma associated with severe orthostatic hypotension. Ann Intern Med 1963; 58: 669 676.

13. Chiappa KH, Young RR. A case of paracarcinomatous pandysautonomia. Neurology (Abstract) 1973; 23: 423.

14. Thomas JP, Shields R. Associated autonomic dysfunction and carcinoma of the pancreas. Br Med J 1970; 4: 32 .

15. Bannister R, Sever R, Gross M. Cardiovascular reflexes and biochemical responses in progressive autonomic failure. Brain 1977; 100: 327-344.

16. Bannister R, Davies B, Holly E, et al. Defective cardiovascular and supersensitivity to sympathomimetic drugs in autonomic failure. Brain 1979; 102: 163-176.

17. Henrich WL. Autonomic insufficiency. Arch Intern Med 1982; 142: 339-344.

18. Dobkin BH, Rosenthal NP. Clinical assessment of autonomic dysfunction: an approach to the Shy-Drager syndrome. Bull Los Angeles Assoc 1975; 3: 101-109.

19. McLeod JG, Tuck RR. Disorders of the autonomic nervous system: Part 2. Investigation and treatment. Ann Neurol 1987; 21: 519529.

20. Anderson NE, Posner JB, Sidtis JJ, et al. The metabolic anatomy of paraneoplastic cerebellar degeneration. Ann Neurol 1988; 23 : 533-540.

21. Ahmed NM, Carpenter $\mathrm{S}$. Autonomic neuropathy and carcinoma of the lung. J. Can Med Assoc 1975; 113: 410-412.

22. Dietl HW, Pulst SM, Engelhardt P, et al. Paraneoplastic brainstem encephalitis with acute dystonia and central hypoventilation. J Neurol 1982; 227: 229-238.

23. Morton DL, Itabashi HH, Grimes OF. Nonmetastatic neurological complications of bronchogenic carcinoma: the carcinomatous neuromyopathies. J Thorac Cardiovasc Surg 1966; 51: 14-28.

24. Polinsky RJ, Kopin IJ, Ebert MH, et al. Pharmacological distinction of different orthostatic hypotension syndromes. Neurology 1981;31:1-7.

25. McLeod JG, Tuck RR. Disorders of the autonomic nervous system: Part 1. Pathophysiology and clinical features. Ann Neurol 1987; 21: 419-430.

26. Mills SE, Walker AN, Cooper PH, et al. Atypical carcinoid tumour of the lung - a clinicopathologic study of 17 cases. Am J Surg Pathol 1982; 6: 643-654. 